\title{
Clinical and neuroimaging characteristics of cerebral sinovenous thrombosis in neonates undergoing cardiac surgery
}

\author{
Nathalie H. P. Claessens, MD, ${ }^{\mathrm{a}, \mathrm{b}, \mathrm{c}}$ Selma O. Algra, MD, PhD, ${ }^{\mathrm{d}}$ Nicolaas J. G. Jansen, MD, PhD, ${ }^{\mathrm{b}}$ \\ Floris Groenendaal, MD, PhD, ${ }^{a}$ Esther de Wit, MD, ${ }^{\mathrm{b}}$ Alexander A. Wilbrink, MD, ${ }^{\mathrm{b}}$ Felix Haas, MD, PhD, ${ }^{\mathrm{e}}$ \\ Antonius N. J. Schouten, MD, ${ }^{\mathrm{f}}$ Rutger A. J. Nievelstein, MD, PhD, ${ }^{\mathrm{d}}$ Manon J. N. L. Benders, MD, PhD, ${ }^{\mathrm{a}}$ and \\ Linda S. de Vries, MD, $\mathrm{PhD}^{\mathrm{a}}$
}

\section{ABSTRACT}

Objectives: Neonates with congenital heart disease may have an increased risk of cerebral sinovenous thrombosis, but incidence rates are lacking. This study describes the clinical and neuroimaging characteristics of cerebral sinovenous thrombosis in neonates undergoing cardiac surgery.

Methods: Forty neonates ( $78 \%$ male) requiring neonatal univentricular or biventricular cardiac repair using cardiopulmonary bypass were included. All underwent preoperative (median postnatal day 7) and postoperative (median postoperative day 7) magnetic resonance imaging of the brain, including venography, to detect cerebral sinovenous thrombosis. Clinical characteristics were compared between cerebral sinovenous thrombosis positive and cerebral sinovenous thrombosis negative neonates.

Results: Postoperatively, cerebral sinovenous thrombosis was diagnosed in 11 neonates $(28 \%)$, with the transverse sinus affected in all, and involvement of multiple sinuses in 10 (91\%). Preoperatively, signs of thrombosis were seen in 3 cases $(8 \%)$. Focal infarction of the basal ganglia was significantly more common in cerebral sinovenous thrombosis positive than cerebral sinovenous thrombosis negative neonates $(P=.025)$. Cerebral sinovenous thrombosis positive neonates spent more time in the intensive care unit preoperatively $(P=.001)$, had lower weight $(P=.024)$ and lower postmenstrual age $(P=.030)$ at surgery, and had prolonged use of a central venous catheter $(P=.023)$ and a catheter placed in the internal jugular vein more often $(P=.039)$. Surgical and postoperative factors were not different between new postoperative cerebral sinovenous thrombosis positive and cerebral sinovenous thrombosis negative neonates.

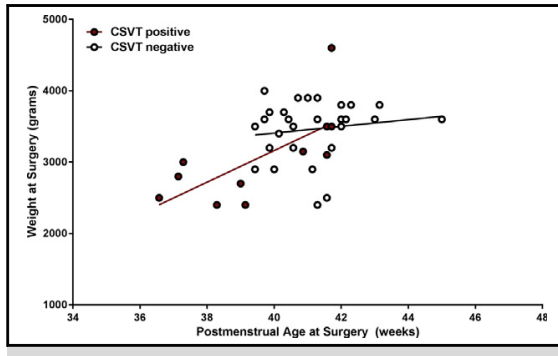

CSVT positive neonates (red) showed lower PMA and weight at surgery.

\section{Central Message}

CSVT might be more common than previously understood in neonates undergoing cardiac surgery.

\section{Perspective}

$\overline{\text { CSVT might be more common around neonatal }}$ cardiac surgery than previously understood. This article describes the clinical and neuroimaging characteristics of CSVT in neonates with CHD. Proposed risk factors include lower PMA and weight at time of surgery, as well as the use of internal jugular vein catheters.

See Editorial Commentary page 1159.

Conclusions: Cerebral sinovenous thrombosis might be more common than previously understood in neonates undergoing cardiac surgery. In our study, cerebral sinovenous thrombosis was associated with a higher risk of additional intraparenchymal brain injury. (J Thorac Cardiovasc Surg 2018;155:1150-8)

\footnotetext{
From the ${ }^{\mathrm{a}}$ Division of Perinatology, Department of Neonatology, ${ }^{\mathrm{b}}$ Division of Pediatrics, Department of Paediatric Intensive Care, ${ }^{c}$ Division of Pediatrics, Department of Pediatric Cardiology, and ${ }^{\mathrm{e}}$ Division of Pediatrics, Department of Pediatric Cardiothoracic Surgery, Wilhelmina Children's Hospital, University Medical Centre Utrecht, Utrecht, The Netherlands; and ${ }^{\mathrm{d}}$ Division of Imaging, Department of Radiology, and ${ }^{\mathrm{f}}$ Department of Anesthesiology, University Medical Centre Utrecht, Utrecht, The Netherlands.

Received for publication Jan 31, 2017; revisions received Sept 28, 2017; accepted for publication Oct 11, 2017; available ahead of print Dec 6, 2017.

Address for reprints: Linda S. de Vries, MD, PhD, Department of Neonatology, University Medical Centre Utrecht, KE 04.123.1, PO Box 85090, 3508 AB Utrecht, The Netherlands (E-mail: 1.s.devries@umcutrecht.nl).

0022-5223/\$36.00

Copyright (c) 2017 by The American Association for Thoracic Surgery https://doi.org/10.1016/j.jtcvs.2017.10.083
}

Major advances in cardiac surgical technique, together with improvements in perioperative intensive care management, have increased the survival of infants with even the most critical forms of congenital heart disease (CHD)

- Scanning this $\mathrm{QR}$ code will take you to a supplemental video for the article.

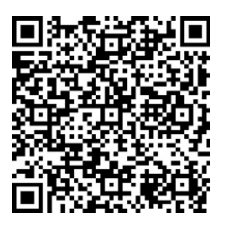



Abbreviations and Acronyms
$\mathrm{BW}=$ birth weight
$\mathrm{CPB}=$ cardiopulmonary bypass
$\mathrm{CHD}=$ congenital heart disease
$\mathrm{CSVT}=$ cerebral sinovenous thrombosis
$\mathrm{CVC}=$ central venous catheter
MRI = magnetic resonance imaging
MRV = magnetic resonance venography
PMA $=$ postmenstrual age

substantially over the past few decades. However, the high rate of altered brain development and structural brain injuries remain reason for concern, especially regarding the serious neurodevelopmental impairments in survivors with CHD. ${ }^{1-4}$

Because of the increased use and sensitivity of neuroimaging in at-risk infants, cerebral sinovenous thrombosis (CSVT) is more often recognized. The incidence of neonatal CSVT is low (estimated at 1-12/100,000 live births), but may have serious consequences for short-term and long-term neurodevelopmental outcome. ${ }^{5,6}$ Outcome studies after neonatal CSVT have shown an increased risk of developmental impairments and epilepsy, especially when CSVT is accompanied by intra-parenchymal brain lesions. ${ }^{6-9}$ Risk factors for CSVT are increasingly reported in the literature, suggesting a multifactorial origin of maternal (preeclampsia, gestational diabetes), perinatal (complicated delivery, asphyxia, prematurity), and neonatal (systemic illness, infection) contributors., ${ }^{5,6}$ Early CSVT diagnosis provides an opportunity to prevent propagation of the thrombus by initiation of anticoagulation therapy. ${ }^{10}$

A previous study from our institution showed that CSVT is a common diagnosis in infants undergoing complex neonatal cardiac surgery. ${ }^{11}$ Generally, the presence of a cardiac malformation is described as a risk factor for the development of CSVT,, 5 but little is known about the characteristics and risk factors of this interaction.

The aim of this study is to describe the clinical and neuroimaging characteristics of CSVT in a cohort of infants with critical CHD undergoing neonatal cardiac repair. The secondary aim is to compare clinical variables and neurodevelopmental outcome parameters between CSVT positive and CSVT negative neonates with CHD.

\section{MATERIALS AND METHODS \\ Study Population}

All neonates with critical CHD born between 2009 and 2016 requiring neonatal cardiac surgery with cardiopulmonary bypass (CPB) at the Wilhelmina Children's Hospital Utrecht, who underwent preoperative and postoperative magnetic resonance imaging (MRI) of the brain (in context of research or standard clinical care) were retrospectively included for this study. The first part of the study population includes a research cohort (2009-2012) of neonates included in a randomized controlled trial comparing the incidence of new postoperative brain injury between 2 intraoperative perfusion techniques. ${ }^{11}$ For this study, 21 eligible neonates were not included (genetic syndrome [ $\mathrm{N}=4]$, logistic problems $[\mathrm{N}=6]$ and parental refusal $[\mathrm{N}=11])$. The research cohort consisted of 37 patients, of whom 8 were excluded for this study because of incomplete MRI data (no T1-weighted imaging or magnetic resonance venography [MRV] $[\mathrm{N}=7]$, or no postoperative MRI [N = 1]). Since 2016, preoperative and postoperative MRI of the brain are part of standard clinical care in neonates undergoing complex cardiac surgery. A total of 11 neonates born between January 2016 and July 2016 were included for this study (5 eligible neonates were not included; died [N=3], emergency surgery without preoperative MRI [ $\mathrm{N}=2])$. The ethical committee approved the previously mentioned randomized controlled trial (Clinical Trial Registration Information http://clinicaltrials.gov; NCT01032876) and gave permission to use the clinically obtained MRI data from January 2016 onwards for research purposes.

\section{Magnetic Resonance Imaging Protocol}

Preoperative MRI was performed the day before surgery or the day of surgery. MRI was performed on a $1.5 \mathrm{~T}$ magnetic resonance system (2009-2012; Gyroscan ACS-NT, Philips Medical Systems, Best, The Netherlands) or a 3.0T magnetic resonance system (from 2013 onwards; Achieva, Philips Medical Systems, Best, The Netherlands). Neonates who required mechanical ventilation at the time of MRI received continuous sedation by morphine, midazolam, and rocuronium. Neonates without mechanical ventilation were fed, swaddled in a vacuum cushion, and if necessary sedated with chloral hydrate (single dose $50-60 \mathrm{mg} / \mathrm{kg}$ ). All neonates received 2 layers of hearing protection. During the MRI oxygen saturation, heart and respiratory rates were monitored. Both 1.5T and 3T MRI protocols included 3-dimensional T1-, T2-, axial diffusion-, and axial susceptibility-weighted sequences. Also, phase-contrast 3-dimensional MRV was performed (1.5T protocol: repetition time $=16 \mathrm{~ms}$, echo time $=6.6 \mathrm{~ms}$, phase contrast velocity $=15 \mathrm{~cm} / \mathrm{s}, 3 T$ protocol: repetition time $=20 \mathrm{~ms}$, echo time $=7.3 \mathrm{~ms}$, phase-contrast velocity $=10 \mathrm{~cm} / \mathrm{s}$ ).

\section{Cerebral Sinovenous Thrombosis and Additional Brain Injury}

All MRI scans were reviewed by a neonatologist and pediatric radiologist with extensive experience in neonatal cerebral imaging, who were blinded for clinical characteristics of the neonates. T1-weighted and MRV images were reviewed for the presence of CSVT. Phase-contrast MRV is known for the high rate of false-positives for neonatal CSVT. In neonates, venous flow velocities are lower than in adults. ${ }^{12,13}$ Slow flow is mainly due to small body size, deformability of the skull, and head position. ${ }^{14,15}$ In addition, all neonates with suspicion of CSVT underwent Doppler ultrasound of the specific sinuses to visualize and describe the thrombus. CSVT was only diagnosed when confirmed on MRV, T1, and ultrasound (Figure 1). Neonates without postoperative T1, MRV, and ultrasound were excluded from this study. MRI scans were also reviewed for presence and severity of white matter injury, (focal) gray matter infarctions, and intraparenchymal hemorrhages.

\section{Perioperative Protocol}

Between birth and surgery, all neonates were admitted to the pediatric cardiology ward or pediatric intensive care unit (in case of need for respiratory or circulatory support). Preoperative preparation of the neonate included placement of a central venous catheter (CVC), preferably placed in the subclavian vein. For neonates who required a new CVC or had an unsuccessful CVC placement in the subclavian vein, the CVC was placed in the internal jugular vein. X-ray was performed to evaluate location and side of CVC directly after placement in all infants.

By standard protocol, all neonates received tranexamic acid $25 \mathrm{mg} / \mathrm{kg}$ in 30 minutes at the start of surgery, with continuation by $10 \mathrm{mg} / \mathrm{kg} / \mathrm{h}$ during the 


\section{Normal MR Venography}
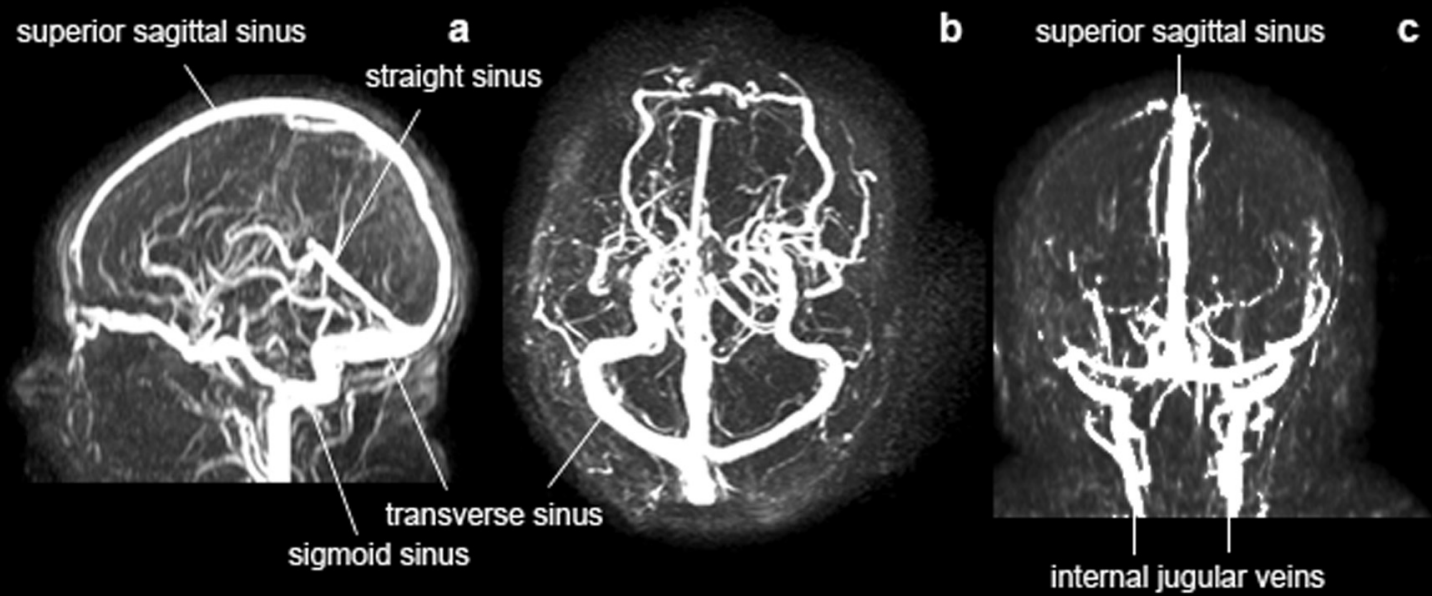

\section{CSVT MR Venography}

a

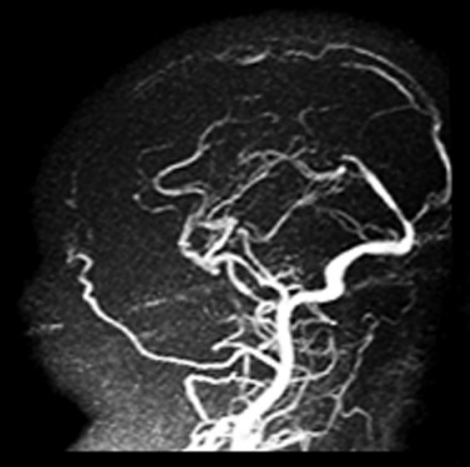

b

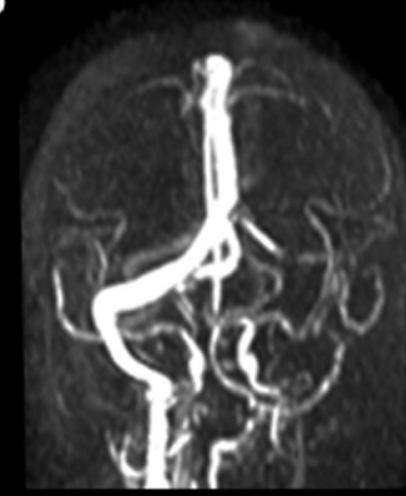

C

\section{CSVT T1 Imaging}
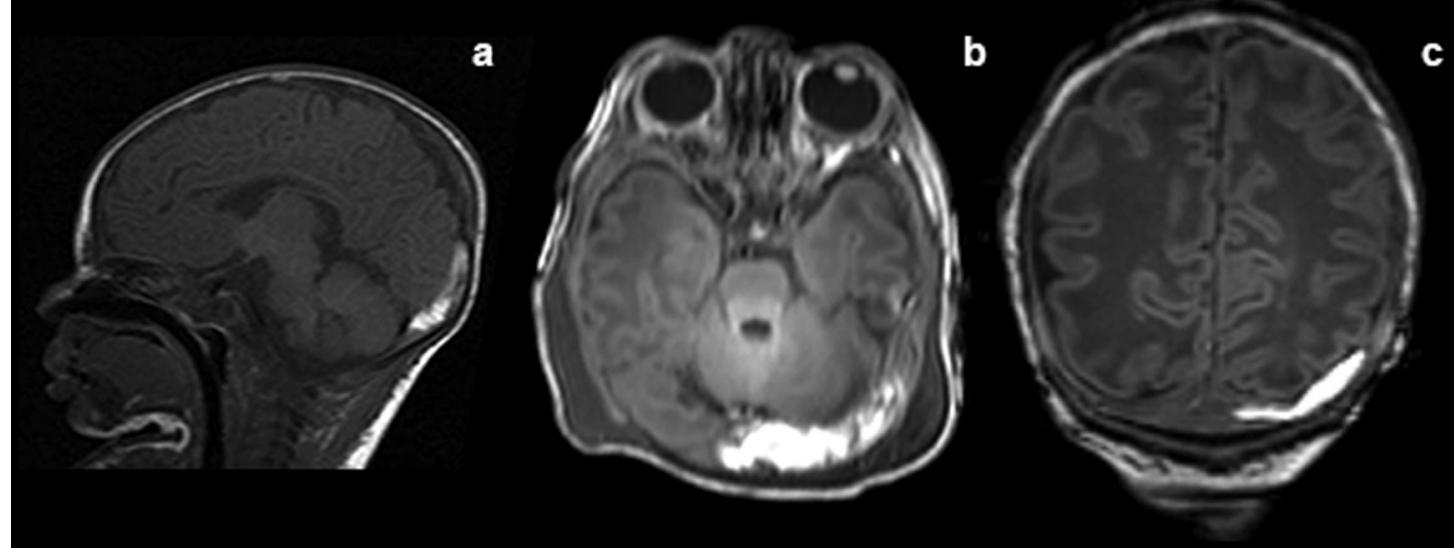

FIGURE 1. In the first row, normal MRV images in (1a) sagittal, (1b) axial, and (1c) coronal view are shown. The cerebral venous drainage system consists of the superficial sinus system (superior sagittal sinus dividing into transverse sinuses and continuing into sigmoid sinuses and internal jugular veins) and deep sinus system (basal veins draining into internal cerebral veins uniting into the inferior sagittal sinus continuing into vein of Galen and straight sinus), which congregate at the base of the superior sagittal sinus. ${ }^{10,24}$ The second row shows 3 neonates with CSVT, with (2a) occlusion of the superior sagittal sinus and straight sinus, (2b) occlusion of the left transverse sinus and sigmoid sinus, and (2c) occlusion of the left transverse sinus, sigmoid sinus, and internal jugular vein. In the third row, the corresponding T1-weighted images of 3 neonates with CSVT are presented with (3a) high signal intensity of the thrombus in superior sagittal sinus in sagittal view, (3b) high signal intensity of the thrombus in the left transverse sinus in axial view, and (3c) high signal intensity of the thrombus in the left transverse sinus in coronal view. MR, Magnetic resonance; CSVT, cerebral sinovenous thrombosis. 
rest of surgery. At the moment of median sternotomy, heparin 3 to $4 \mathrm{mg} / \mathrm{kg}$ was administered with administration of protamine sulfate 3 to $4 \mathrm{mg} / \mathrm{kg}$ at the end of surgery. Activated clotting time was frequently measured during surgery ( $>440$ seconds aimed) and postoperatively at the intensive care (100-160 seconds aimed, with extra admission of protamine sulfate if necessary). Neonates undergoing aortic arch surgery were cooled to a nasopharyngeal temperature of approximately $18^{\circ} \mathrm{C}$. Neonates undergoing an arterial switch procedure or truncus arteriosus correction were cooled to approximately $28^{\circ} \mathrm{C}$. Antegrade cerebral perfusion or deep hypothermic cardiac arrest was chosen as the perfusion technique by standard double venous cannulation. In neonates who underwent Norwood surgery or shunt placement, postoperative prophylactic anticoagulation therapy (heparin $10 \mathrm{IE} /$ $\mathrm{kg} / \mathrm{h}$ ) was initiated as soon as drain production was less than $0.5 \mathrm{~mL} / \mathrm{kg} / \mathrm{h}$.

\section{Clinical Variables}

Postmenstrual age (PMA) at birth was measured from the first day of last menstruation. Birth weight $(\mathrm{BW}) z$ scores were measured using the Dutch neonatal reference charts. ${ }^{16}$ Cardiac risk categories and RiskAdjusted Congenital Heart Surgery scores were calculated. ${ }^{17}$ Because the number of days between birth and surgery were variable, preoperative time spent at the intensive care and on mechanical ventilation was calculated in percentage of total time between birth and surgery. Regarding the postoperative period, number of days at the intensive care and on mechanical ventilation were measured. Infection was defined as a positive blood culture in combination with clinical signs of infection (temperature instability, decreased $\left[<4000 / \mathrm{mm}^{3}\right]$ or elevated $\left[>12,000 / \mathrm{mm}^{3}\right]$ white blood cell count). Only postoperative infections diagnosed before the postoperative MRI were taken into account. Preoperative requirement of furosemide (diuretics) was reviewed. Because most neonates received furosemide in the first 72 hours after surgery, dosage of furosemide $(\mathrm{mg} / \mathrm{kg})$ was also calculated. Postoperative transfusions of erythrocytes, platelets, and fresh-frozen plasma were reviewed $(\mathrm{mL} / \mathrm{kg})$. None of the neonates received cryoprecipitate or specific anticoagulation agents (eg, prothrombin complex). All neonates were monitored with continuous amplitude-integrated electroencephalography during the first 48 to 72 hours after surgery to diagnose electrographic (subclinical) seizures. Neurodevelopmental outcome at the age of 2 years was measured using the Dutch Bayley Score of Infant and Toddler Development, Third Edition (Bayley-III-NL, 2006; Amsterdam, Pearson Assessment and Information BV). Outcome assessment was available in 26 children (did not yet reach age of $2[\mathrm{~N}=11]$, lost to follow-up $[\mathrm{N}=2]$, died $[\mathrm{N}=1])$.

\section{Statistical Analysis}

Statistical analysis was performed using IBM SPSS Statistics version 21.0 (New York, NY). An independent $t$ test, Mann-Whitney $U$ test, or Fisher exact test was used to examine the difference in clinical variables between CSVT positive and CSVT negatives neonates depending on type of variable and normality of distribution. Patient characteristics and preoperative and perioperative factors were compared between all CSVT positive and CSVT negative neonates. Fluid balances and surgical and postoperative factors were compared between new postoperative CSVT positive and CSVT negative neonates. Univariable linear regression analysis was used to explore the relation between PMA and weight at surgery. Bivariate logistic regression analysis was performed for the continuous clinical variables that were significant in the $t$ test analysis with calculation of $P$ value, odds ratio (OR), and $95 \%$ confidence interval. Because of the small sample size, no multivariable logistic regression analysis was performed.

\section{RESULTS}

Forty neonates with critical CHD (diagnoses are shown in Table 1) were included, in whom both preoperative
TABLE 1. Included cardiac diagnoses $(N=40)$

\begin{tabular}{lc}
\hline RACH-S category 3 & $6(15 \%)$ \\
TGA & 4 \\
Coarctation of the aorta & 2 \\
RACH-S category 4 & $23(57 \%)$ \\
Hypoplastic left heart complex & 5 \\
TGA + VSD & 1 \\
Interrupted or hypoplastic aortic arch & 2 \\
+ ASD or VSD & 7 \\
+ TGA & 2 \\
+ TGA and double-outlet right ventricle & 5 \\
Truncus arteriosus & 1 \\
RACH-S category 6 & $11(28 \%)$ \\
SVP left-sided obstruction & 9 \\
SVP right-sided obstruction & 2 \\
\hline
\end{tabular}

$R A C H-S$, Risk Adjustment for Congenital Heart Surgery; TGA, transposition of the great arteries; $V S D$, ventricular septal defect; $A S D$, atrial septal defect; $S V P$, single ventricle physiology.

(median postnatal day 7) and postoperative (median postoperative day 7) MRI were performed. Baseline characteristics are presented in Table 2.

\section{Cerebral Sinovenous Thrombosis Characteristics}

Eleven infants (28\%) were diagnosed with CSVT at postoperative MRI. Preoperatively, signs of thrombosis were seen in 3 neonates $(8 \%)$, but all without complete obstruction of the sinus(es). In all with preoperative signs, the thrombosis had worsened at postoperative MRI to complete obstruction. As shown in Figure 2, the transverse sinus was involved in all $(100 \%)$ with continuation into the sigmoid sinus in $7(64 \%)$ and the jugular vein in $2(18 \%)$. Occlusion of the superior sagittal sinus was seen in $5(45 \%)$ and the straight sinus in $1(9 \%)$. In 10 CSVT positive cases, multiple sinuses were involved $(91 \%)$. Representative images are provided in Figure 1.

\section{Comparison of Clinical Characteristics}

At birth, PMA and weight were not significantly different between CSVT positive and CSVT negative neonates (Table 2). One CSVT positive neonate was very large for gestation (BW $z$ score 2.78) due to maternal gestational diabetes. For BW $z$ score, larger deviations from zero were seen in CSVT positive neonates (median $|0.85|$ vs $|0.45|$, $P=.020)$ than in CSVT negative neonates, showing that CSVT positive neonates are relatively small and large for gestation. CSVT positive cases spent relatively more preoperative time at the intensive care unit (mean 5 of 8 days vs 3 of 12 days, $P=.001$ ), with nonsignificantly more time on mechanical ventilation (mean 3 of 8 days vs 2 of 12 days) when compared with CSVT negative neonates.

Linear regression analysis showed a significant relationship between PMA and weight at surgery in CSVT positive neonates $\left(\mathrm{R}^{2} 0.485, P .02\right)$ but not in CSVT negative 
TABLE 2. Comparison clinical characteristics

\begin{tabular}{|c|c|c|c|c|c|}
\hline Patient characteristics & $\begin{array}{l}\text { CSVT negative } \\
\quad(N=29)\end{array}$ & $\begin{array}{l}\text { CSVT positive } \\
\quad(\mathrm{N}=11)\end{array}$ & $P$ value & $\begin{array}{c}\text { New postoperative } \\
\text { CSVT positive }(N=8)\end{array}$ & $P$ value \\
\hline PMA at birth (wk)* & $39.1(38.8-40.1)$ & $39.3(37.6-40.4)$ & .353 & & \\
\hline $\mathrm{BW}(\mathrm{g})^{*}$ & $3400(3258-3635)$ & $3030(2600-3440)$ & .137 & & \\
\hline BW $z$ score* & $-0.09(-0.59-0.19)$ & $-0.7(-1.3-0.3)$ & .698 & & \\
\hline Deviation BW $z$ score from $0^{*}$ & $0.45(0.12-0.67)$ & $0.85(0.46-1.44)$ & .020 & & \\
\hline Male $\dagger$ & $22(76)$ & $9(82)$ & .523 & & \\
\hline Prenatal diagnosis $\dagger$ & $18(62)$ & $9(82)$ & .211 & & \\
\hline RACHS* & $28.6(24.8-46.9)$ & $28.6(28.6-41.9)$ & .840 & & \\
\hline \multicolumn{6}{|l|}{ Preoperative course } \\
\hline Balloon atrioseptostomy $\dagger$ & $4(14)$ & $2(18)$ & .536 & & \\
\hline$\%$ of time at mechanical ventilation $*$ & $0(0-17)$ & $14(0-80)$ & .241 & & \\
\hline$\%$ of time at Intensive care* & $22(13-35)$ & $67(40-92)$ & .001 & & \\
\hline Infection $\dagger$ & $5(17)$ & $2(18)$ & .636 & & \\
\hline Furosemide $\dagger$ & $14(48)$ & $6(55)$ & .500 & & \\
\hline \multicolumn{6}{|l|}{ Perioperative course } \\
\hline Univentricular repair $\dagger$ & $9(31)$ & $2(18)$ & .349 & & \\
\hline Postnatal age at surgery $(\mathrm{d})^{*}$ & $9(8-15)$ & $9(7-10)$ & .139 & & \\
\hline PMA at surgery $(w k)^{*}$ & $41.1(40.1-42.0)$ & $39.1(37.3-41.5)$ & .030 & & \\
\hline Weight at surgery $(\mathrm{g})^{*}$ & $3600(3200-3750)$ & $3000(2500-3500)$ & .024 & & \\
\hline Surgery weight - BW $(\mathrm{g})^{*}$ & $100(-10-183)$ & $-20(-100-10)$ & .002 & & \\
\hline $\mathrm{CVC} \mathrm{d}^{*}$ & $7(3-9)$ & $8(6-13)$ & .023 & & \\
\hline CVC in internal jugular vein $\dagger$ & $2(7)$ & $4(36)$ & .039 & & \\
\hline \multicolumn{6}{|l|}{ Surgery } \\
\hline Duration surgery $(\mathrm{h})^{*}$ & $4.7(4.4-5.5)$ & & & $4.8(4.2-5.6)$ & .662 \\
\hline Duration CPB (min)* & $159(132-185)$ & & & $153(136-215)$ & .849 \\
\hline Lowest rectal temperature $\left({ }^{\circ} \mathrm{C}\right)^{*}$ & $22(20-24)$ & & & $20(19-23)$ & .244 \\
\hline Lowest hematocrit value during surgery $(\mathrm{vol} \%)^{*}$ & $24(23-26)$ & & & $26(24-27)$ & .110 \\
\hline \multicolumn{6}{|l|}{ Postoperative course } \\
\hline Activated clotting time end surgery $(\mathrm{sec}) \mathrm{N}=38^{*}$ & $144(126-154)$ & & & $131(117-138)$ & .458 \\
\hline Extra admission protamine sulfate $\dagger$ & $9(31)$ & & & $0(0)$ & .081 \\
\hline Platelet count, mean first $24 \mathrm{~h}^{*}$ & $191(148-234)$ & & & $218(200-241)$ & .070 \\
\hline Hematocrit, mean first $24 \mathrm{~h}\left(\mathrm{vol}_{\mathrm{o}}\right)^{*}$ & $37(34-41)$ & & & $39(36-41)$ & .841 \\
\hline Postoperative mechanical ventilation (total days)* & $5(3-8)$ & & & $6(4-12)$ & .967 \\
\hline Postoperative intensive care stay (total days)* & $7(5-12)$ & & & $7(6-15)$ & .734 \\
\hline Infection $\dagger$ & $2(7)$ & & & $0(0)$ & 1.000 \\
\hline Furosemide, first $72 \mathrm{~h} \dagger$ & $27(93)$ & & & $8(100)$ & 1.000 \\
\hline Dosage furosemide, first $72 \mathrm{~h}$ (total $\mathrm{mg} / \mathrm{kg}$ )* & $46(26-85)$ & & & $34(25-44)$ & .429 \\
\hline Erythrocyte transfusions, first $72 \mathrm{~h} \dagger$ & $20(69)$ & & & $4(50)$ & .413 \\
\hline Platelet transfusions, first $72 \mathrm{~h} \dagger$ & $5(17)$ & & & $1(13)$ & 1.000 \\
\hline Fresh-frozen plasma transfusions, first $72 \mathrm{~h} \dagger$ & $11(38)$ & & & $5(63)$ & .254 \\
\hline Heparin administration, first $72 \mathrm{~h} \dagger$ & $13(45)$ & & & $3(38)$ & 1.000 \\
\hline Central venous pressure, first $24 \mathrm{~h}(\text { mean } \mathrm{cm} \mathrm{H} 2 \mathrm{O})^{*}$ & $10.0(8.2-11.9)$ & & & $13(8-14)$ & .352 \\
\hline \multicolumn{6}{|l|}{ Fluid balance $(\mathrm{mL}, \text { per } 24 \mathrm{~h})^{*}$} \\
\hline Preoperative day $\mathrm{N}=31$ & $27(11-44)$ & & & $158(65-239)$ & .427 \\
\hline Day of surgery & $22(13-53)$ & & & $78(32-226)$ & .555 \\
\hline Postoperative day 1 & $34(1-60)$ & & & $204(91-295)$ & .117 \\
\hline Postoperative day 2 & $-17(-52-3)$ & & & $42(-137-154)$ & .230 \\
\hline Postoperative day 3 & $-11(-41-1)$ & & & $-7(-268-39)$ & .947 \\
\hline
\end{tabular}

Comparison of clinical characteristics between CSVT negative and CSVT positive neonates with CHD. Fluid balances and surgical and postoperative factors were compared between new postoperative CSVT positive and CSVT negative neonates. Bold indicates a $P$ value $<.05$. CSVT, Cerebral sinovenous thrombosis; $P M A$, premenstrual age; $B W$, birth weight; RACHS, Risk Adjustment Congenital Heart Surgery Score; $C V C$, central venous catheter; $C P B$, cardiopulmonary bypass. *Median (interquartile range). $\dagger \mathrm{N}(\%)$. 


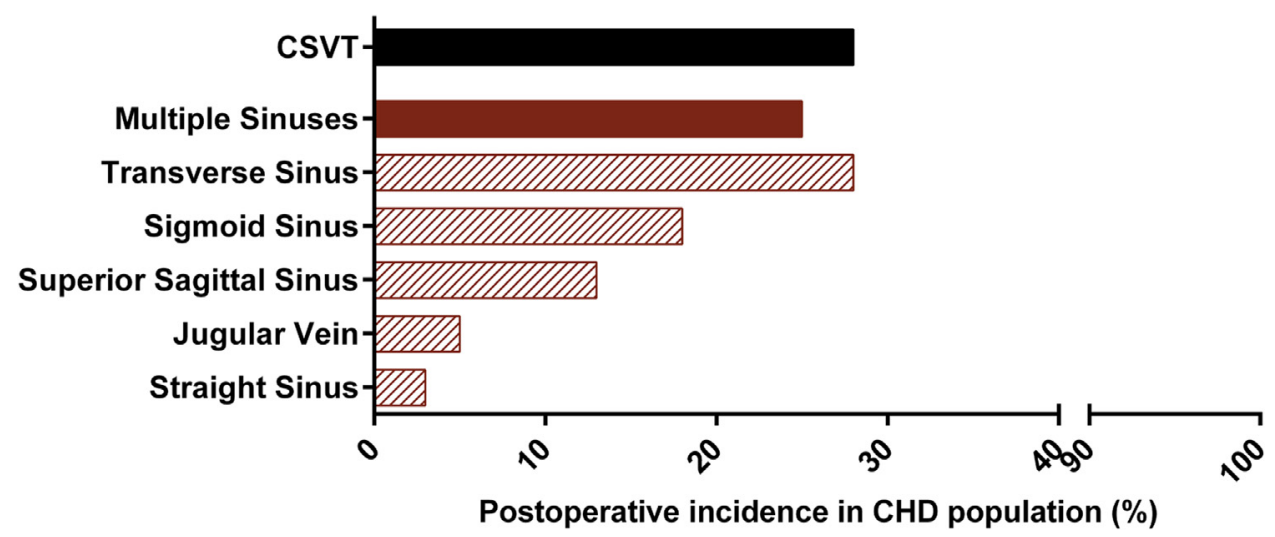

FIGURE 2. Eleven neonates (28\%) showed CSVT at the postoperative MRI. Affected sinuses (y-axis) with frequency of involvement (x-axis) at postoperative timepoint are shown by the graph bars. CSVT, Cerebral sinovenous thrombosis; CHD, congenital heart disease.

neonates $\left(\mathrm{R}^{2} 0.02, P=.446\right.$, Figure 3$)$. However, CSVT positive neonates had lower PMA at surgery (median 39.1 vs 41.1 weeks, $P=.03$ ) than CSVT negative neonates, as well as a lower weight (median 3000 vs $3600, P$.024). Also, CSVT positive cases had not yet regained their BW at surgery $(-20 \mathrm{~g})$ when CSVT negative neonates had $(+100 \mathrm{~g}, P=.002)$.

CSVT positive cases had prolonged presence of a CVC (median 8 vs 7 days, mean 10 vs 6 days, $P .023$ ), with a CVC placed in the internal jugular vein more often compared with CSVT negative neonates (4/11 vs $2 / 29$, $P .039)$. In 9 of 11 patients ( $82 \%$ ), the side of CVC corresponded with the (most severely) affected side of CSVT (P.083).

Comparison of surgical and postoperative factors between neonates with new postoperative CSVT positive neonates (so excluding 3 neonates with signs of thrombosis preoperatively) and CSVT negative neonates did not show any differences between the 2 groups (Table 2).

Bivariate logistic regression analysis (Table 3) showed significant relationships between CSVT development and

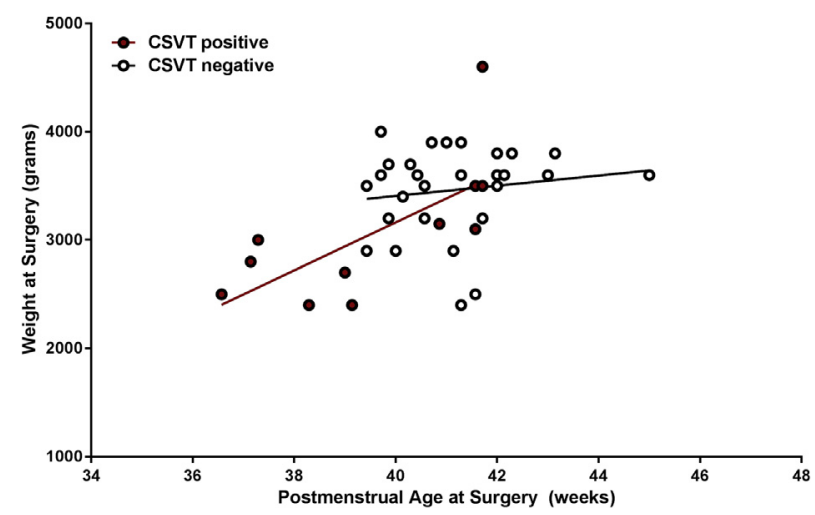

FIGURE 3. Association of PMA (x-axis, in weeks) and weight at surgery (y-axis, in grams) for CSVT positive (red dots) and CSVT negative neonates (open dots). CSVT, Cerebral sinovenous thrombosis. preoperative time at the intensive care unit (OR, 1.043) and duration of CVC (OR, 1.206), and inverse relationships between CSVT and PMA at surgery (OR, 0.485), as well as weight at surgery (OR, 0.844).

\section{Neurologic Injury and Neurodevelopment}

Nine $(82 \%)$ CSVT positive neonates showed severe new postoperative brain lesions, compared with $21(72 \%)$ of the CSVT negative neonates $(P=.696)$ (Table 4). Focal infarction of the basal ganglia or thalamus was significantly more common in the CSVT positive group than the CSVT negative group $(5 / 11$ vs $3 / 29, P=.025)$. There was no difference in incidence of moderate-severe white matter injury between CSVT positive and CSVT negative neonates (6/11 vs 20/29). Postoperatively, electrographic seizures were seen in 4 CSVT positive neonates $(36 \%)$ versus 7 CSVT negative neonates $(24 \%, P=.345)$. All neonates with electrographic seizures showed new postoperative brain lesions. Anticoagulation therapy was initiated after confirmation of CSVT in all. Follow-up MRI at the age of 3 months was performed in 10 of 11 CSVT positive cases, and none of these infants had developed new intraparenchymal lesions. No differences were seen in mean motor or cognitive composite score between CSVT positive and CSVT negative neonates (Table 4). Neonates with a basal ganglia or thalamus infarction showed lower motor scores than infants without (mean 93 vs $103, P=.08)$. Three neonates $(7.5 \%)$ were diagnosed

TABLE 3. Bivariate logistic regression analysis

\begin{tabular}{lccc}
\hline \multicolumn{1}{c}{ Clinical risk factor } & $\boldsymbol{P}$ & OR & $\mathbf{9 5} \% \mathbf{C I}$ \\
\hline $\begin{array}{l}\text { Time at intensive care } \\
\text { preoperative }(\%)\end{array}$ & .004 & 1.043 & $1.014-1.073$ \\
PMA at surgery (wk) & .013 & 0.485 & $0.274-0.857$ \\
Weight at surgery (100 g) & .032 & 0.844 & $0.723-0.985$ \\
Duration CVC (d) & .035 & 1.206 & $1.013-1.434$ \\
\hline
\end{tabular}

Dependent variable: presence of CSVT. $O R$, Odds ratio; $C I$, confidence interval $P M A$, postmenstrual age; $C V C$, central venous catheter. 
TABLE 4. Neurologic injury and development

\begin{tabular}{|c|c|c|c|}
\hline Postoperative brain injury & CSVT negative $(N=29)$ & CSVT positive $(N=11)$ & $P$ \\
\hline Postnatal age at preoperative MRI (d)* & $7(6-12)$ & $6(4-8)$ & .135 \\
\hline Postoperative age at postoperative MRI (d)* & $6(5-8)$ & $7(6-9)$ & .511 \\
\hline New postoperative moderate-severe brain injury $\dagger$ & $21(72)$ & $9(82)$ & .696 \\
\hline Moderate-severe white matter injury $\dagger$ & $20(69)$ & $6(55)$ & .469 \\
\hline Focal infarction Basal Ganglia $\dagger$ & $3(10)$ & $5(46)$ & .025 \\
\hline Subclinical postoperative seizures $\dagger$ & $7(24)$ & $4(36)$ & .345 \\
\hline \multicolumn{4}{|l|}{ Neurodevelopment $\mathrm{N}=26$} \\
\hline Age at BSITD-III $(\mathrm{y})^{*}$ & $2.0(2.0-2.1)$ & $2.0(2.0-2.1)$ & .965 \\
\hline BSITD-III cognitive composite score* & $100(90-113)$ & $98(91-104)$ & .354 \\
\hline BSITD-III motor composite score* & $103(88-113)$ & $100(100-103)$ & .534 \\
\hline Cerebral palsy $\dagger$ & $2(7)$ & $1(9)$ & .652 \\
\hline
\end{tabular}

Comparison of MRI brain injury and neurodevelopmental outcome at the age of 2 years between CSVT negative and CSVT positive neonates. Bold indicates a $P$ value $<.05$. CSVT, Cerebral sinovenous thrombosis; MRI, magnetic resonance imaging; BSITD, Bayley Score of Infant and Toddler Development. *Median (interquartile range). $\dagger \mathrm{N}$ (\%).

with cerebral palsy (gross motor function classification system level $1[\mathrm{~N}=2]$ and level $2[\mathrm{~N}=1])$, of whom 1 was also diagnosed with CSVT.

\section{DISCUSSION}

This study showed that CSVT might be more common than previously understood in neonates undergoing cardiac surgery for critical CHD and examined the clinical and neuroimaging characteristics of CSVT in this population (Video 1). Differences in perioperative and surgical management likely contribute to variability in CSVT incidence and characteristics between centers.

Focal infarctions of the basal ganglia and thalamus were more frequent in neonates with postoperative CSVT than without, and children with this type of infarction showed lower motor scores at the age of 2 years. In previous work on CSVT, involvement of multiple sinuses was described to be associated with both venous and arterial (hemorrhagic) infarctions of the basal ganglia and thalamus. ${ }^{6,9,18-20} \mathrm{In}$ case of CSVT, venous pressure increases in the occluded vessels causing brain tissue venous congestion, vasogenic edema, and increased capillary pressure leading to secondary (hemorrhagic) infarction. ${ }^{21}$ Future research might focus on the coexistence of CSVT with intraparenchymal infarctions and whether these injuries are caused by the same (prothrombotic) determinants.

In our population, electrographic seizures were as frequent in CSVT positive as in CSVT negative neonates, likely because of the similar overall incidences of brain injury. ${ }^{22}$ Neonatal seizures are often reported as a first symptom of neonatal CSVT, but a broad spectrum of subtle and nonspecific clinical signs can be seen. ${ }^{5,6,9,21}$ Most of these signs (lethargy, poor feeding, changes in muscle tone) are hard to assess in postoperatively sedated neonates, which underlines the importance of early appropriate neuroimaging and neuromonitoring after neonatal cardiac surgery.

All neonates with critical CHD are exposed to the CPB circuit in early life, and this in itself might be a risk factor for the development of CSVT. CPB takes over cardiac and pulmonary function during cardiac surgery using external membrane oxygenation to exchange oxygen and carbon dioxide, with both arterial and venous cannulas placed in the patient. Because of the large size of the cannulas and changes in intravenous pressure and embolisms, the endothelial wall of the venous system can be damaged during $\mathrm{CPB}$, activating the local coagulation system. ${ }^{23,24}$ Extracorporeal membrane oxygenation, a different form of extracorporeal life support but similar to $\mathrm{CPB}$, was predictive of CSVT in several previous studies in both neonates and children. ${ }^{6,18}$ In our study, time spent on CPB during cardiac surgery was not different between CSVT positive and CSVT negative neonates. Future studies with

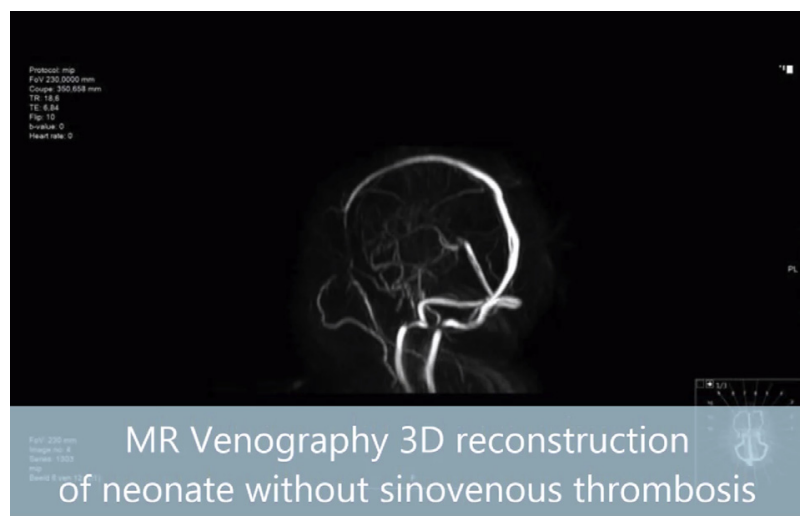

VIDEO 1. This video presents the clinical and neuroimaging characteristics of CSVT in neonates undergoing cardiac surgery. Video available at: http://www.jtcvsonline.org/article/S0022-5223(17)32426-1/fulltext. 
inclusion of neonates undergoing (cardiac) surgery without the use of CPB should elucidate this effect further.

Previous research has shown that hypercoaguable and prothrombotic state increases the risk of CSVT development in infancy and childhood. ${ }^{5,25}$ Subjects in our study all underwent the same perioperative coagulation protocol with standard use of heparin and protamine sulfate. Postoperatively, protamine sulfate was only administered when activated clotting time remained above reference value $(>160)$. Remarkably, none of the neonates who required extra postoperative protamine sulfate developed CSVT. A recent study underlined the challenges of anticoagulation therapy in neonates, because there is a wide range of baseline antithrombin levels in neonates. ${ }^{26}$ Lower baseline antithrombin activity is associated with lower efficacy of heparin administered around cardiac surgery. We speculate that differences in maturity of the neonatal coagulation system (and thus response to [anti] coagulant medication) might play an important role in the development of CSVT after neonatal cardiac surgery.

Longer use of a CVC was seen in neonates who developed postoperative CSVT, and in the majority, the CVC side corresponded with the most affected side of CSVT. A CVC can induce endothelial damage of the vessel wall, which is associated with (locally) elevated coagulation factors. ${ }^{23}$ Also, CVC entails a risk of line-associated thrombosis. In this study, we showed that a CVC placed in the internal jugular vein (instead of the subclavian or femoral vein) increased the risk of CSVT. This finding is an expected one, because the internal jugular vein is part of the cerebral venous circulation. However, it should be considered that placement of a CVC in the internal jugular vein was not the location of first choice in our population, but reserved for children who required a second CVC or had an unsuccessful CVC placement in the subclavian vein. We recommend to avoid placement of the CVC in the internal jugular vein in neonates undergoing cardiac surgery, and if placement in the internal jugular vein is necessary, prophylactic initiation of anticoagulation therapy should be considered, as well as in case of prolonged CVC requirement.

No differences in neurodevelopmental outcome at the age of 2 years were seen between CSVT positive and negative children. In the literature, prognosis of CSVT seems highly dependent on the extent of additional brain lesions. $^{5,9,27}$ In our study, all CSVT positive neonates received anticoagulation therapy (low-molecular-weight heparin) for 3 months (with follow-up MRI at that age). Lack of anticoagulation therapy is predictive of clot propagation in neonatal CSVT, ${ }^{27,28}$ which is accompanied with higher rates of additional brain injury. In our population, none of the neonates with CSVT developed (new) intraparenchymal hemorrhages after initiation of anticoagulation therapy.
This study is the first to describe neuroimaging and clinical characteristics of CSVT in a well-defined population of neonates undergoing cardiac surgery. This study shows that neonates with critical CHD are prone to develop CSVT. However, it should be mentioned that our population might not reflect CHD populations from other centers, because perioperative management and $\mathrm{CPB}$ strategies are likely to affect the incidence of CSVT.

\section{Study Limitations}

Limitations of the study include the relatively small sample size, because only subjects with preoperative and postoperative MRI of the brain were included. The exclusion of neonates with incomplete MRI data might influence the results, although no differences in baseline characteristics were seen between inclusions and exclusions. 3T MRI was used in neonates with CHD from 2013 onward, whereas before that time $1.5 \mathrm{~T}$ imaging was the standard imaging modality for this patient group. However, slice thickness of all sequences were similar, and no differences in presence of CSVT or additional brain lesions were seen between neonates scanned with the $1.5 \mathrm{~T}$ or $3 \mathrm{~T}$ MRI protocol. Our study design did not allow analysis of the timing of onset of CSVT, because no imaging of the brain was performed between surgery and postoperative MRI of the brain. By the retrospective design of this study, it was not possible to investigate the role of coagulation system maturity in the development of CSVT.

\section{CONCLUSIONS}

From this study, we conclude that CSVT might be more common than previously understood in neonates undergoing cardiac surgery for critical CHD (Video 1). Proposed risk factors include lower PMA and weight at time of surgery, as well as the use of internal jugular vein catheters. Incidences likely vary between centers because of differing perioperative management and surgical strategies. In our study, CSVT was associated with higher risk of additional intraparenchymal brain injury. Early diagnosis of CSVT allows early initiation of anticoagulation therapy, helping to prevent additional brain injury.

\section{Conflict of Interest Statement}

Authors have nothing to disclose with regard to commercial support.

The authors thank the Congenital Heart Disease Life Span Study Group of the Wilhelmina Children's Hospital, which includes the Department of Neonatology, Department of Pediatric Intensive Care, Department of Pediatric Cardiology, Department of Pediatric Cardiothoracic Surgery, Department of Pediatric Anesthesiology, Department of Obstetrics, Department of Radiology, Department of Medical Psychology, and Child Development and Exercise Centre. 


\section{References}

1. Miller SP, McQuillen PS, Hamrick S, Xu D, Glidden DV, Charlton N, et al. Abnormal brain development in newborns with congenital heart disease. $N$ Engl J Med. 2007;357:1928-38.

2. McQuillen PS, Miller SP. Congenital heart disease and brain development. Ann N Y Acad Sci. 2010;1184:68-86.

3. Sarajuuri A, Jokinen E, Puosi R, Eronen M, Mildh L, Mattila I, et al. Neurodevelopmental and neuroradiologic outcomes in patients with univentricular heart aged 5 to 7 years: related risk factor analysis. J Thorac Cardiovasc Surg. 2007; 133:1524-32.

4. International Cardiac Collaborative on Neurodevelopment I. Impact of operative and postoperative factors on neurodevelopmental outcomes after cardiac operations. Ann Thorac Surg. 2016;102:843-9.

5. deVeber G, Andrew M, Adams C, Bjornson B, Booth F, Buckley DJ, et al. Cerebral sinovenous thrombosis in children. N Engl J Med. 2001;345:417-23.

6. Berfelo FJ, Kersbergen KJ, van Ommen CH, Govaert P, van Straaten HL, PollThe BT, et al. Neonatal cerebral sinovenous thrombosis from symptom to outcome. Stroke. 2010;41:1382-8.

7. Kersbergen KJ, Groenendaal F, Benders MJ, van Straaten HL, Niwa T, Nievelstein RA, et al. The spectrum of associated brain lesions in cerebral sinovenous thrombosis: relation to gestational age and outcome. Arch Dis Child Fetal Neonatal Ed. 2011;96:F404-9.

8. Grunt S, Wingeier K, Wehrli E, Boltshauser E, Capone A, Fluss J, et al. Cerebral sinus venous thrombosis in Swiss children. Dev Med Child Neurol. 2010;52: $1145-50$.

9. Fitzgerald KC, Williams LS, Garg BP, Carvalho KS, Golomb MR. Cerebral sinovenous thrombosis in the neonate. Arch Neurol. 2006;63:405-9.

10. Kersbergen KJ, Groenendaal F, Benders MJ, de Vries LS. Neonatal cerebral sinovenous thrombosis: neuroimaging and long-term follow-up. J Child Neurol. 2011;26:1111-20.

11. Algra SO, Jansen NJ, van der Tweel I, Schouten AN, Groenendaal F, Toet M, et al. Neurological injury after neonatal cardiac surgery: a randomized, controlled trial of 2 perfusion techniques. Circulation. 2014;129:224-33.

12. Taylor GA. Intracranial venous system in the newborn: evaluation of normal anatomy and flow characteristics with color Doppler US. Radiology. 1992;183: 449-52.

13. Fenton AC, Papathoma E, Evans DH, Levene MI. Neonatal cerebral venous flow velocity measurement using a color flow Doppler system. J Clin Ultrasound. 1991;19:69-72.

14. Widjaja E, Shroff M, Blaser S, Laughlin S, Raybaud C. 2D time-of-flight MR venography in neonates: anatomy and pitfalls. AJNR Am J Neuroradiol. 2006; 27:1913-8.

15. Tan M, Deveber G, Shroff M, Moharir M, Pontigon AM, Widjaja E, et al. Sagittal sinus compression is associated with neonatal cerebral sinovenous thrombosis. Pediatrics. 2011;128:e429-35.
16. Visser GH, Eilers PH, Elferink-Stinkens PM, Merkus HM, Wit JM. New Dutch reference curves for birthweight by gestational age. Early Hum Dev. 2009;85: $737-44$.

17. Jenkins KJ, Gauvreau K, Newburger JW, Spray TL, Moller JH, Iezzoni LI. Consensus-based method for risk adjustment for surgery for congenital heart disease. J Thorac Cardiovasc Surg. 2002;123:110-8.

18. Wu YW, Miller SP, Chin K, Collins AE, Lomeli SC, Chuang NA, et al. Multiple risk factors in neonatal sinovenous thrombosis. Neurology. 2002;59:438-40.

19. Mullins ME, Grant PE, Wang B, Gonzalez RG, Schaefer PW. Parenchymal abnormalities associated with cerebral venous sinus thrombosis: assessment with diffusion-weighted MR imaging. AJNR Am J Neuroradiol. 2004;25:1666-75.

20. Sebire G, Tabarki B, Saunders DE, Leroy I, Liesner R, Saint-Martin C, et al. Cerebral venous sinus thrombosis in children: risk factors, presentation, diagnosis and outcome. Brain. 2005;128(Pt 3):477-89.

21. Yang JY, Chan AK, Callen DJ, Paes BA. Neonatal cerebral sinovenous thrombosis: sifting the evidence for a diagnostic plan and treatment strategy. Pediatrics. 2010;126:e693-700.

22. Algra SO, Schouten AN, Jansen NJ, van Oeveren W, Haas F, Groenendaal F, et al. Perioperative and bedside cerebral monitoring identifies cerebral injury after surgical correction of congenital aortic arch obstruction. Intensive Care Med. 2015; 41:2011-2.

23. Riccabona M, Kuttnig-Haim M, Dacar D, Urlesberger B, Reiterer F, Maurer U, et al. Venous thrombosis in and after extracorporeal membrane oxygenation: detection and follow-up by color Doppler sonography. Eur Radiol. 1997;7: 1383-6.

24. Teksam M, Moharir M, Deveber G, Shroff M. Frequency and topographic distribution of brain lesions in pediatric cerebral venous thrombosis. AJNR Am J Neuroradiol. 2008;29:1961-5.

25. Wasay M, Dai AI, Ansari M, Shaikh Z, Roach ES. Cerebral venous sinus thrombosis in children: a multicenter cohort from the United States. J Child Neurol. 2008;23:26-31.

26. Manlhiot C, Gruenwald CE, Holtby HM, Brandao LR, Chan AK, Van Arsdell GS, et al. Challenges with heparin-based anticoagulation during cardiopulmonary bypass in children: Impact of low antithrombin activity. J Thorac Cardiovasc Surg. 2016;151:444-50.

27. Moharir MD, Shroff M, Pontigon AM, Askalan R, Yau I, Macgregor D, et al. A prospective outcome study of neonatal cerebral sinovenous thrombosis. $J$ Child Neurol. 2011;26:1137-44.

28. Moharir MD, Shroff M, Stephens D, Pontigon AM, Chan A, MacGregor D, et al. Anticoagulants in pediatric cerebral sinovenous thrombosis: a safety and outcome study. Ann Neurol. 2010;67:590-9.

Key Words: congenital heart disease, MRI, brain, newborn, venography 